\title{
Technical note: A novel boundary condition using contact elements for finite element based deformable image registration
}

\author{
Tiezhi Zhang, Nigel P. Orton, T. Rockwell Mackie, and Bhudatt R. Paliwal \\ Department of Human Oncology, University of Wisconsin, Madison, Wisconsin 53792
}

\begin{abstract}
(Received 13 January 2004; revised 28 May 2004; accepted for publication 1 June 2004;
\end{abstract} published 13 August 2004)

\begin{abstract}
Deformable image registration is an important tool for image-guided radiotherapy. Physics-modelbased deformable image registration using finite element analysis is one of the methods currently being investigated. The calculation accuracy of finite element analysis is dependent on given boundary conditions, which are usually based on the surface matching of the organ in two images. Such a surface matching, however, is hard to obtain from medical images. In this study, we developed a new boundary condition to circumvent the traditional difficulties. Finite element contact-impact analysis was employed to simulate the interaction between the organ of interest and the surrounding body. The displacement loading is not necessarily specified. The algorithm automatically deforms the organ model into the minimum internal energy state. The analysis was performed on CT images of the lung at two different breathing phases (exhalation and full inhalation). The result gave the displacement vector map inside the lung. Validation of the result showed satisfactory agreement in most parts of the lung. This approach is simple, operator independent and may provide improved accuracy of the prediction of organ deformation. (C) 2004 American Association of Physicists in Medicine. [DOI: 10.1118/1.1774131]
\end{abstract}

\section{INTRODUCTION}

Deformable image registration (DIR) or nonrigid image registration provides voxel-voxel matching information between mono- or multi-modality medical images. It is of importance for adaptive radiotherapy or image-guided radiotherapy. ${ }^{1-6}$ Currently DIR may be classified into two categories: image based and physics-model-based. Image-based DIR uses image information, such as landmarks or intensities, to find the best voxel matching between two images. Physics-modelbased DIR considers the organ as an elastic object and studies its deformation by finite element analysis (FEA). ${ }^{7-11}$ The accuracy of physics-model-based DIR is determined by how well the model simulates the real situation.

A finite element model includes material properties, geometry, and loadings. For DIR purposes, usually displacement loadings are applied directly to the surface or at inner feature point positions. Displacements of anatomical landmarks, such as blood vessel bifurcations and organ surface corners, can be measured directly from the CT images. However, the number of well-defined landmarks that can be distinguished from medical images is limited. An insufficient number of loading points in the model may lead to uncertainties in the results. Some studies deform a surface elastically first in order to match the surfaces, then use the surface displacement to deform the body. ${ }^{10,11}$ This approach does not accurately simulate the actual physics of organ deformation and may lead to errors even if the accurate elasticity information is provided.

In this study, we used finite element contact-impact analysis to circumvent the difficulty of obtaining matched feature points. This work is similar to a study by Liang et al. who used an iterative algorithm to search for the minimum internal elastic energy state while maintaining the organ shape. ${ }^{8}$
However, our method is straightforward and does not require special FEA programming.

\section{MATERIAL AND METHODS}

\section{A. Principle}

During normal breathing, the pleural cavity volume increases by the downward movement of the diaphragm and outward movement of the ribs. The pleural cavity pressure decreases whereas the inside lung pressure remains the same as outer atmosphere. Lung volume expands under the negative surface pressure. The lung tightly occupies the pleural cavity all the time.

This process is simulated using finite element contactimpact analysis. ${ }^{12}$ Contact between the lung and thoracic wall is modeled using contact elements. Figure 1 shows the diagram of the contact element and the solid element. The contact surfaces are meshed with contact source elements and contact target elements. The rigidity of the surface is adjusted by the parameters of the contact elements. Surface contact is detected via penetration of the contact elements, and pressure is applied on the surface based on the penetration. As the surface pressure gradually decreases, the lung volume expands until it fully occupies the volume enclosed by the contact target surface. The voxel displacements are interpolated from the displacements of the nodes.

\section{B. Image acquisition}

Lung images at the exhalation phase and deep inspiration phase were obtained by breath holding CT scans. A spirometer (Medgraphics Corp, St. Paul, MN) was used to record breathing signal during the CT scans. Using a GE LightSpeed CT scanner with four row detectors (GE Medical System, Waukesha, WI), the scan at deep inspiration phase took 


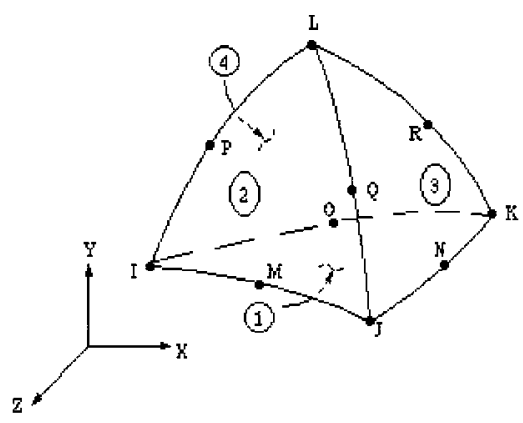

(a)

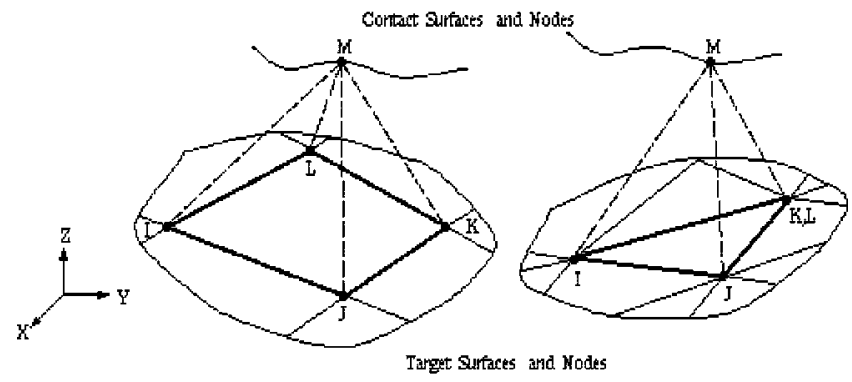

(b)

FIG. 1. Diagram of solid and contact elements used in this study (ANSYS Inc.). (a) Ten node tetrahedral solid element. (b) Contact elements.

about $20 \mathrm{~s}$ with $0.8 \mathrm{~s}$ gantry rotation speed. Holding breath at exhalation phase was extremely difficult for the patients. The scan at exhalation phase was scanned with $0.4 \mathrm{~s}$ gantry rotation speed and finished within $10 \mathrm{~s}$. Figure 2 shows the patient's breathing curve during CT scans.

\section{Finite element model}

The lungs were contoured from the CT images at two phases using Pinnacle ${ }^{\mathrm{TM}}$ commercial treatment planning software (Philips Radiation Oncology Systems, Madison, WI). The surfaces were reconstructed from the point clouds using FastRBF software (Farfield Technology, Christchurch, New

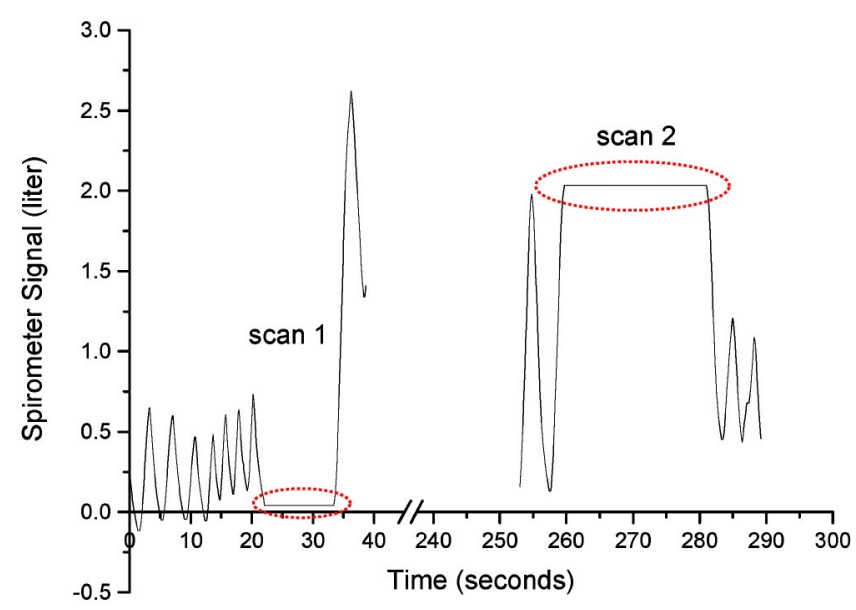

FIG. 2. Breath holding lung CT images were obtained at two different breathing phases, exhalation and deep inspiration phases. The patient's breathing was monitored by a spirometer. With calibration, the spirometer signal reflects the total lung volume changes.

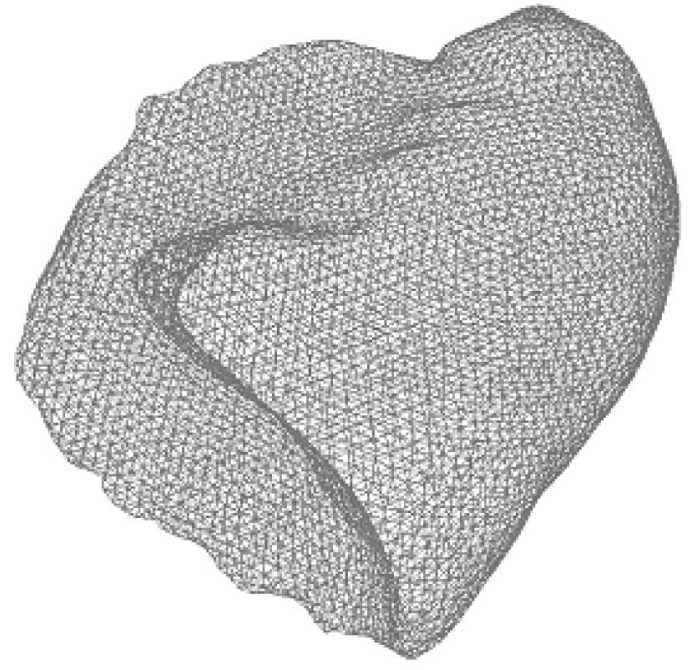

FIG. 3. A lung surface reconstructed from CT images.

Zealand). Figure 3 shows a reconstructed lung surface represented by a triangle mesh. The lung surfaces were then imported to the finite element analysis software ANSYS (ANSYS Inc., Canonsburg, PA). The solid lung model was created using the surface of the lung at exhalation phase and meshed with ten-node tetrahedral solid elements. The surface of the solid model was then meshed with contact source elements and the lung surface at inspiration phase was meshed with the contact target surface. A total of 9085 tetrahedral solid elements, 2584 contact source elements and 1692 contact target elements was used in creating the model.

The constitutive equation of lung tissue elasticity can be found in Refs. 12-16. There is no consensus in the values in the literature. In this pilot study, for simplicity reasons, the lung was modeled as linear isotopic elastic material with Young's modulus $\mathrm{E}=4 \mathrm{kPa}$ and Poisson's ratio $\gamma=0.35$. Numerous options and parameters are available for contact elements. Standard contact without friction was used in this study. An important factor affecting the solution convergence rate is the normal contact stiffness factor FKN. After several trials, we found a FKN value in the range of $0.1-1$ was able to give the optimal convergence rate and acceptable penetration.

\section{Solution}

For contact-impact analysis of models with complex shape such as the lung, the ANSYS large displacement solver, which uses an iterative algorithm, offers the fastest solution. Without using the large displacement solver, the solution sometimes fails to converge even if the deformation is small.

Zero-displacement constraints were assigned on the nodes of the contact target elements. Negative surface pressure was gradually applied to the solid lung surface. Lung volume expanded due to the negative surface pressure until it filled the space enclosed by the contact target surface. 

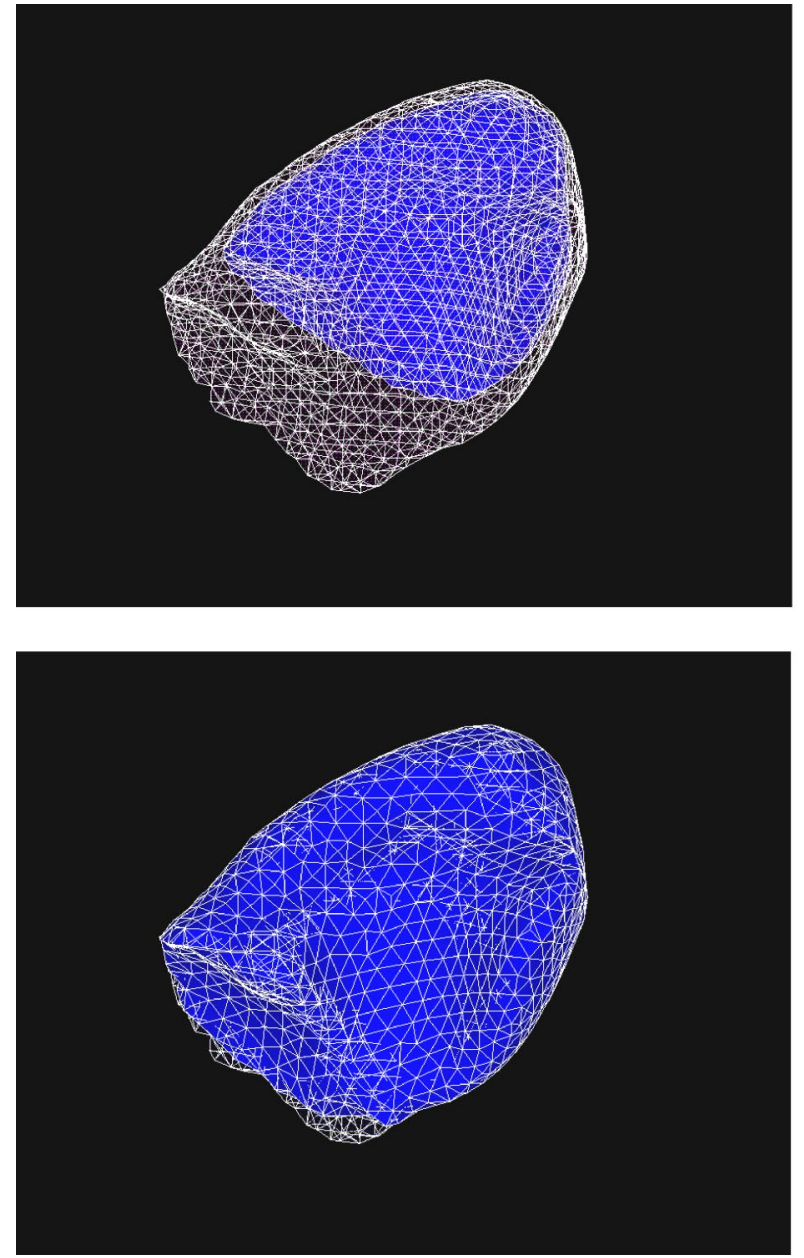

FIG. 4. The initial state (a) and ending state (b) of finite element contactimpact analysis. The transparent mesh is the contact target surface extracted from the images of the lung at inhalation phase; the inner mesh is the solid lung model created from the exhalation lung images. After simulation, the solid lung model occupied the space enclosed by the surface of the lung at inhalation phase.

\section{RESULTS}

Figure 4 shows the initial and ending states of this process. At the initial state, empty space existed between the inner solid lung surface and outer contact target surface. At the ending state, most of the space was occupied by the expanded lung model. A small amount of space was still left at the edge of the lung, which was most likely caused by segmentation uncertainties.

FEA gave the displacements at nodal positions, and the voxel displacements were interpolated from the results at surrounding nodes. Figure 5 shows the displacement vector map of the lung. The magnitude of motion varied for different anatomical positions. The largest displacement of the lung from exhalation to deep inhalation occurred at the lower lobe with a magnitude as large as $6 \mathrm{~cm}$.

For the purpose of validation, an image of the lung at exhalation phase was also obtained by 3-D image warping, using the displacement maps and the lung image at inhalation phase. In principle, the image obtained from image warping should be the same as the image obtained directly

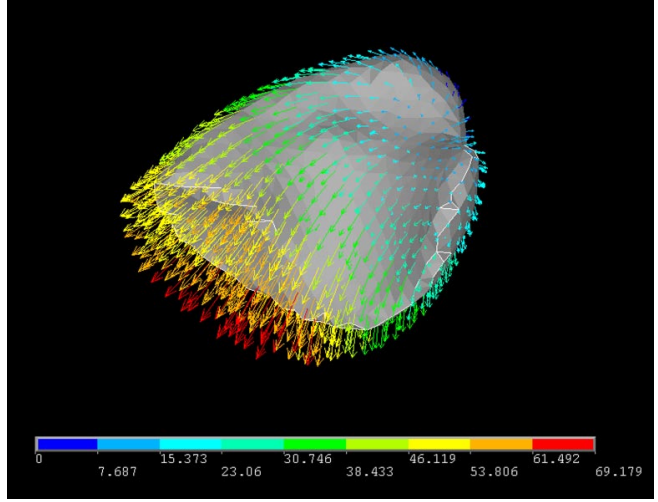

FIG. 5. The displacement vectors given by finite element analysis. The results of DIR show the voxel displacement changing with anatomical position. The lower lobe of the lung exhibits the largest displacement.

from the CT scan. Figure 6 shows the overlay of the two images. Good agreement is obtained, especially at the lower lobe of the lung. A small amount of discrepancy can be observed at the top of the lung.

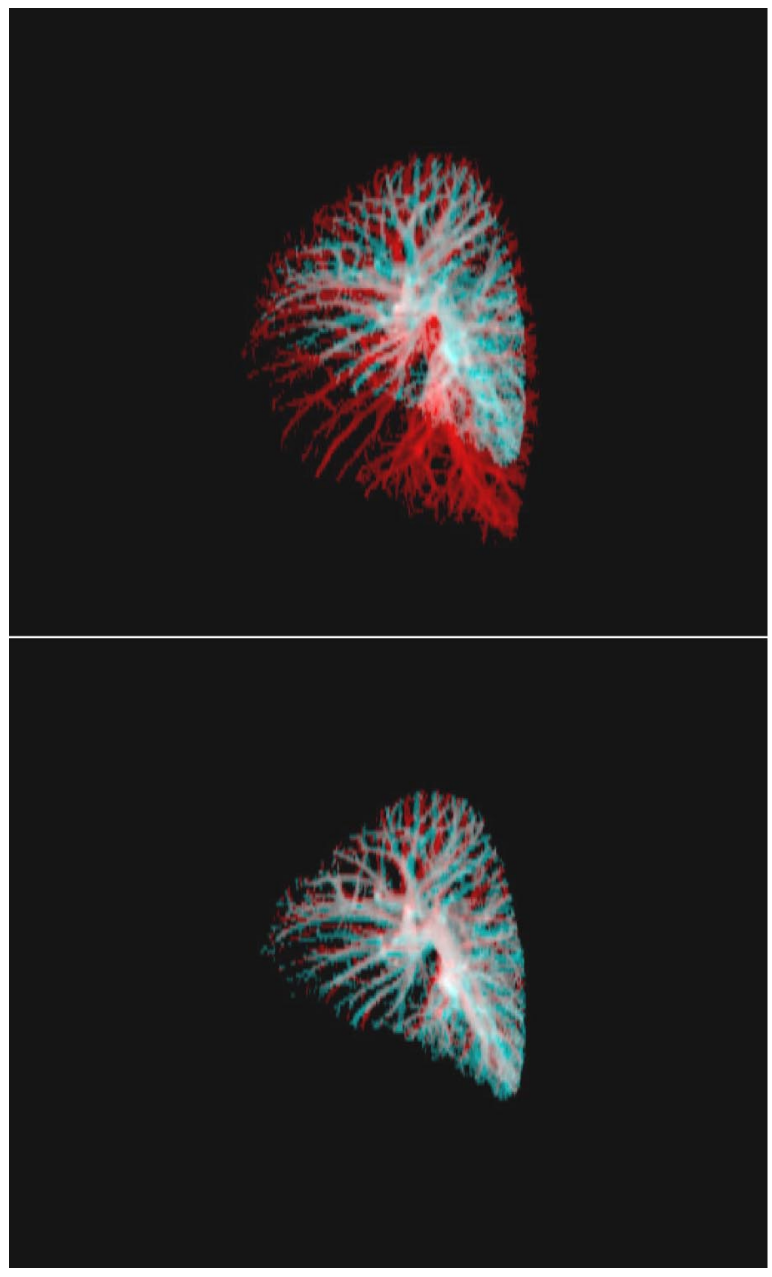

(a)

(b)

FIG. 6. The validation of DIR results by image warping: (a) Compares the images of the lung at inhalation phase and exhalation phase. Both are directly obtained from $\mathrm{CT}$ scans and (b) shows the images of the lung at exhalation phase obtained by image warping and direct CT scan. 


\section{DISCUSSION}

Contact-impact analysis mimics the interaction between an organ and surrounding body parts. It potentially provides more accurate prediction of organ deformation. This method may also be applied to other anatomical sites such as the liver and prostate. Only organ boundary image information is needed for such physics-model-based DIR, so this method has the fewest requirements on image quality and can be employed with other imaging modalities, such as ultrasound and PET, for which image noise makes image-based DIR difficult.

Besides boundary conditions, the material elasticity model is an important factor that determines the accuracy. Living tissue usually shows complex elasticity properties. ${ }^{16}$ For small deformations, nonlinear material properties may be approximated by a linear isotropic elasticity model. However, the simplified material model may lead to errors in modeling of large deformation.

After flow calibration, ${ }^{17}$ the spirometer measures the total volume change of both lungs, which was about $2 l$ for this particular case. The volume of the studied lung was calculated using the surface mesh by

$$
V=\frac{1}{6} \sum_{n} \vec{x}_{n 1} \times \vec{x}_{n 2} \cdot \vec{x}_{n 3},
$$

where $V$ is the volume of the lung; $n$ is the number of triangle facets; and $\vec{x}_{n 1}, \vec{x}_{n 2}, \vec{x}_{n 3}$ are the vectors to the triangle vertices. $\vec{x}_{n 1}, \vec{x}_{n 2}, \vec{x}_{n 3}$ are in right-hand order with normal pointing out of the volume. The lung volume changed from 3.2 to $4.0 l$ and increased by about $25 \%$. For this range of deformation, lung tissue may exhibit nonlinear properties. This may be the reason for the observed discrepancy seen at the top of the lung in Fig. 6. ANSYS has the capacity to simulate nonlinear hyperelastic or viscoelastic materials, which may better model the living tissues.

Finite element contact-impact analysis is a computationally expensive process. In this study, modeling took about 3 to $4 \mathrm{~h}$ on a dual $1.8 \mathrm{GHz}$ Intel CPU Dell workstation. In spite of the drawback, finite element analysis with the contact element boundary is easy to use, and it will be a valuable tool in developing new treatment techniques that require deformable image registration.

\section{ACKNOWLEDGMENT}

This work was partially supported by NIH Grant No. P01 CA-88960.

${ }^{1}$ C. Wu, R. Jeraj, W. Lu, and T. R. Mackie, "Fast treatment plan modification with an over-relaxed Cimmino algorithm," Med. Phys. 31, 191200 (2004).

${ }^{2}$ C. Wu, R. Jeraj, G. H. Olivera, and T. R. Mackie, "Re-optimization in adaptive radiotherapy," Phys. Med. Biol. 47, 3181-3195 (2002).

${ }^{3}$ W. Lu, "Motion detection and correction for image guided radiation therapy," Ph.D. thesis. Medical physics department, University of Wisconsin-Madison, 2001.

${ }^{4}$ D. Yan, F. Vicini, J. Wong, and A. Martinez, "Adaptive radiation therapy," Phys. Med. Biol. 42, 123-132 (1997).

${ }^{5}$ H. Keller, M. A. Ritter, and T. R. Mackie, "Optimal stochastic correction strategies for rigid-body target motion," Int. J. Radiat. Oncol., Biol., Phys. 55, 261-270 (2003).

${ }^{6}$ T. R. Mackie, J. Kapatoes, K. Ruchala, W. Lu, C. Wu, C. Olivera, L. Forrest, W. Tome, J. Welsh, R. Jeraj, P. Harari, P. Reckwerdt, B. Paliwal, M. Ritter, H. Keller, J. Fowler, and M. Metha, "Image guidance for precise conformal radiotherapy," Int. J. Radiat. Oncol., Biol., Phys. 56, 89-105 (2003).

${ }^{7}$ K. K. Brock, S. J. Hollister, L. A. Dawson, and J. M. Balter, "Technical note: Creating a four-dimensional model of the liver using finite element analysis," Med. Phys. 29, 1403-1405 (2002).

${ }^{8} \mathrm{~J}$. Liang and D. Yana, "Reducing uncertainties in volumetric image-based deformable organ registration," Med. Phys. 30, 2116-2122 (2003).

${ }^{9}$ A. Bharatha, M. Hirose, N. Hata, S. K. Warfield, M. Ferrant, K. H. Zou, E. Suarez-Santana, J. Ruiz-Alzola, A. D'Amico, R. A. Cormack, R. Kikinis, F. A. Jolesz, and C. M. Tempany, "Evaluation of three-dimensional finite element-based deformable registration of pre- and intraoperative prostate imaging," Med. Phys. 28, 2551-2560 (2001).

${ }^{10}$ M. K. Chung, K. J. Worsley, S. Robbins, T. Paus, J. Taylor, J. N. Giedd, J. L. Rapoport, and A. C. Evans, "Deformation-based surface morphometry applied to gray matter deformation," NeoroImage 18, 198-213 (2003).

${ }^{11}$ M. Ferrant, A. Nabavi, B. Macq, F. A. Jolesz, R. Kikinis, and S. K. Warfield, "Registration of 3-D intraoperative MR images of the brain using a finite-element biomechanical model," IEEE Trans. Med. Imaging 20, 1384-1397 (2001).

${ }^{12}$ Z. H. Zhong, Finite Element Procedures for Contact-impact Problems (Oxford University Press, New York, 1993).

${ }^{13}$ D. L. Vawter, Y. C. Fung, and J. B. West, "Constitutive equation of lung tissue elasticity," J. Biomech. Eng. 101, 39-45 (1979).

${ }^{14}$ Y. Lanir, "Constitutive equation for the lung tissue," J. Biomech. Eng. 105, 374-380 (1983).

${ }^{15}$ Y. J. Zeng, D. Yager, and Y. C. Fung, "Measurement of the mechanical properties of the human lung tissue," J. Biomech. Eng. 109, 169-174 (1987).

${ }^{16}$ Y. C. Fung, Biomechanics: Mechanical Properties of Living Tissues, 2nd ed. (Springer-Verlag, New York, 1993).

${ }^{17}$ T. Zhang, H. Keller, M. O'Brien, T. R. Mackie, and B. Paliwal, “Application of the spirometer in respiratory gated radiotherapy," Med. Phys. 30, 3165-3172 (2003). 